\title{
Whole-blood RNA transcript-based models can predict clinical response in two large independent clinical studies of patients with advanced melanoma treated with the checkpoint inhibitor, tremelimumab
}

Philip Friedlander ${ }^{1 *}$ (D, Karl Wassmann ${ }^{2}$, Alan M. Christenfeld ${ }^{2}$, David Fisher ${ }^{3}$, Chrisann Kyi ${ }^{1}$, John M. Kirkwood ${ }^{4}$, Nina Bhardwaj, ${ }^{1,5}$ and William K. Oh ${ }^{1}$

\begin{abstract}
Background: Tremelimumab is an antibody that blocks CTLA-4 and demonstrates clinical efficacy in a subset of advanced melanoma patients. An unmet clinical need exists for blood-based response-predictive gene signatures to facilitate clinically effective and cost-efficient use of such immunotherapeutic interventions.

Methods: Peripheral blood samples were collected in PAXgene ${ }^{\oplus}$ tubes from 210 treatment-naïve melanoma patients receiving tremelimumab in a worldwide, multicenter phase III study (discovery dataset). A central panel of radiologists determined objective response using RECIST criteria. Gene expression for 169 mRNA transcripts was measured using quantitative PCR. A 15-gene pre-treatment response-predictive classifier model was identified. An independent population ( $N=150)$ of refractory melanoma patients receiving tremelimumab after chemotherapy enrolled in a worldwide phase II study (validation dataset). The classifier model, using the same genes, coefficients and constants for objective response and one-year survival after treatment, was applied to the validation dataset.

Results: A 15-gene pre-treatment classifier model (containing ADAM17, CDK2, CDKN2A, DPP4, ERBB2, HLA-DRA, ICOS, ITGA4, LARGE, MYC, NAB2, NRAS, RHOC, TGFB1, and TIMP1) achieved an area under the curve (AUC) of 0.86 ( $95 \%$ confidence interval 0.81 to $0.91, p<0.0001$ ) for objective response and 0.6 ( $95 \%$ confidence interval 0.54 to $0.67, p=0.0066)$ for one-year survival in the discovery set. This model was validated in the validation set with AUCs of 0.62 (95\% confidence interval 0.54 to $0.70 p=0.0455$ ) for objective response and 0.68 for one-year survival (95\% confidence interval 0.59 to $0.75 p=0.0002$ ).
\end{abstract}

Conclusions: To our knowledge, this is the largest blood-based biomarker study of a checkpoint inhibitor, tremelimumab, which demonstrates a validated pre-treatment mRNA classifier model that predicts clinical response. The data suggest that the model captures a biological signature representative of genes needed for a robust anti-cancer immune response. It also identifies non-responders to tremelimumab at baseline prior to treatment.

Keywords: Melanoma, Biomarker, Tremelimumab, CTLA-4, Predictive

\footnotetext{
* Correspondence: philip.friedlander@mssm.edu

'Division of Hematology and Medical Oncology, Tisch Cancer Institute, Icahn

School of Medicine at Mount Sinai, Mount Sinai Hospital, New York, NY, USA

Full list of author information is available at the end of the article
} 


\section{Background}

The value of immunotherapy in treating stage IV melanoma became indisputable in 2011, when the U.S. Food and Drug Administration approved ipilimumab, a CTLA-4 inhibitor. CTLA-4 expressed on the surface of activated T cells binds to B7 (CD80) on antigen-presenting cells with higher affinity than the co-stimulatory protein CD28. Disrupting B7's binding to CD28 prevents T-cell costimulation, leading to dampening of the immune response. Ipilimumab is an IgG1 monoclonal antibody that binds CTLA-4 in an inhibitory manner. Treating relapsed or refractory stage IV melanoma with ipilimumab confers an overall survival benefit versus peptide vaccine treatment, with median survival increasing from 6 to 10 months and 2 -year survival increasing from 14 to $24 \%$ [1]. The actual treatment response rate approximates $10 \%$ [1]. Benefit is durable, with the overall survival rate plateauing at $21 \%$ by year 3 and follow-up that now extends beyond year 10 [2].

Tremelimumab is an IgG2 monoclonal antibody that also inhibits CTLA-4. In a phase II trial in treatmentrefractory metastatic melanoma, an objective response rate of $6.6 \%$ was observed [3]. A subsequent open label phase III trial in treatment-naïve patients demonstrated no survival benefit of tremelimumab compared with chemotherapy, although clinical efficacy was noted in a subset of patients (with a $10.7 \%$ objective response rate). Notably, the duration of response was significantly longer in responders to immunotherapy (35.8 vs. 13.7 months) [4]. Tremelimumab is currently undergoing further clinical investigation.

Disrupting other immune checkpoints, such as the interaction of programmed cell death protein-1 (PD-1) with its ligand PD-L1 can lead to clinical benefit. Melanoma cells express PD-L1, which binds to PD-1 on infiltrating T-cells, leading to decreased T-cell activity. Two PD-1 inhibitors, nivolumab and pembrolizumab, are FDA-approved for treating advanced melanoma, with response rates approximating 40\% and 5-year survival post-nivolumab treatment of 35\% [5-7]. Recently, concurrent CTLA-4 and PD-1 inhibition (ipilimumab plus nivolumab) was FDA-approved on the basis of a $57.6 \%$ response rate [8].

The development of antibodies which inhibit CTLA-4 and PD-1 have improved the efficacy of treatment available to patients with stage IV melanoma. While treatment benefits a subset of patients, many do not respond. Identifying biomarkers which reliably identify treatment responders and long term survivors would allow for early selection of alternative treatment in patients unlikely to benefit, while limiting toxicity risk and health care expenditures. Of patients treated with ipilimumab monotherapy, PD-1 inhibitor monotherapy, or ipilimumab plus nivolumab combination therapy, $27 \%, 17 \%$ and 55\%, respectively, develop high-grade toxicities [8]. The annual regimen price per patient in the US is approximately
$\$ 120,000$ for ipilimumab and $\$ 150,000$ for either of the two PD-1 inhibitors. The combination of ipilimumab plus nivolumab costs approximately $\$ 256,000$ per patient. The Centers for Medicare \& Medicaid Services has reimbursed most of these immunotherapy costs over the past two years, however, European reimbursement rates are far lower: France, the UK, Germany and Italy reimburse approximately $55 \%, 40 \%, 30 \%$, and $30 \%$ of the cost, respectively [9]. Development of a pretreatment biomarker predictive of efficacy to anti-CTLA4 or anti-PD1 blockade would allow for selection of patients more likely to respond to treatment and guide efficacious treatment selection. Expensive immunotherapies could be selectively recommended to patients likely to respond. This would not only prevent patients unlikely to respond from developing toxicity to ineffective therapy but also could lower cost to the health care system through decreased spending on expensive but ineffective therapy.

Discovery of biomarkers robustly predictive of treatment efficacy prior to actually initiating treatment should optimize treatment planning, as well as limit toxicity risk and healthcare expenditure. Still, no tissue- or blood-based biomarker is approved to select melanoma patients for immunotherapy. While a tissue-based biomarker, PD-L1 IHC 28-8 pharmDx (Dako), is commercially available, data do not support its ability to determine prior to treatment which patients will respond to anti-PD-1 immunotherapy [10]. Melanoma patients with low PD-L1 $(<5 \%)$ tumor expression have 41.3 and $54.8 \%$ response rates to nivolumab and combined nivolumab plus ipilimumab treatment, respectively [11].

We reexamined data of 169 mRNA-based transcripts from whole blood collected prior to treatment with tremelimumab in melanoma patients in two large, independent clinical trials. Previously, we reported a whole blood 4-gene mRNA signature was predictive for overall survival in melanoma patients treated with tremelimumab. We demonstrated that CTSD, PLA2G7, TXNRD1, and IRAK3 expression levels in peripheral blood predict overall survival [12]. However this signature did not predict for response to tremelimumab.

The current analysis uses a novel computational systems biology approach, exploiting more recent knowledge of the mechanism of checkpoint inhibition. We proceeded with the hypothesis that whole blood RNA transcript-based genes predictive for both objective response and survival could be identified pre-treatment in advanced melanoma patients treated with tremelimumab. We define a pretreatment mRNA gene signature obtained from blood that predicts response.

\section{Methods}

\section{Patient population}

The patient population in Table 1 has been previously described [12]. Both the discovery and validation 
Table 1 Demographics of patients in the discovery and validation populations

\begin{tabular}{lll}
\hline & Discovery data set & Validation data set \\
\hline Number of patients & 210 & 150 \\
Age, median (range) years & $59(22-90)$ & $53(18-89)$ \\
Gender n (\%) & $117(56 \%)$ & $94(63 \%)$ \\
Male & $93(44 \%)$ & $56(37 \%)$ \\
Female & & \\
Objective Response n (\%) & $28(13 \%)$ & $130(87 \%)$ \\
Patient responsive & $182(87 \%)$ & \\
Patients non-responsive & & $43(29 \%)$ \\
One-year Survival n (\%) & $118(56 \%)$ & $107(71 \%)$ \\
Patient alive at one year & $92(44 \%)$ & Yes \\
Patient deceased at one & & \\
year & No & $58(59 \%)$ \\
Prior chemotherapy & & $5(3 \%)$ \\
Stage of disease n (\%) & $13(6 \%)$ & $16(11 \%)$ \\
IIIC & $35(17 \%)$ & $30(20 \%)$ \\
IV M1A & $49(23 \%)$ & $99(66 \%)$ \\
IV M1B & $113(54 \%)$ & \\
IV M1C & $166(79 \%)$ & \\
Live in United States n (\%) & & \\
U.S. & & \\
Non-U.S. & & \\
\hline
\end{tabular}

datasets resulted from pre-treatment blood samples collected in multinational, open-label studies of tremelimumab administered to advanced melanoma patients. Only patients from whom both a pre- and a post-treatment blood sample were available were included in our analysis. The pre-treatment discovery dataset was a randomized phase III study which treated 325 patients with tremelimumab of which 210 patients had both a pre- and a posttreatment blood sample available with $N=28$ responders and $N=182$ non-responders [4]. The pre-treatment validation dataset was a non-randomized phase IIb study which enrolled 251 patients of which 150 patients had both a pre- and a post-treatment blood sample available with $N=20$ responders and $N=130$ non-responders [3]. The phase IIb study initially reported responses in 16 patients but subsequent central radiology review determined that responses developed in 20 patients. Response results for the phase III study were also determined by central radiologist review of imaging. For our analysis response determination was based upon the results of the central radiology reviews. In both studies, response was determined by RECIST criteria [13]. The patients in the pretreatment discovery dataset were treatment-naïve, while the patients in the pre-treatment validation dataset were chemotherapy-refractory. Therefore, the pre-treatment discovery dataset patients had a longer median survival of 13 months compared with the validation dataset patients at 8.8 months. Similarly, one-year survival for the discovery and validation datasets was 56 and 29\%, respectively.

\section{Gene selection and sample processing}

The selection process for the 169 genes tested has been described previously and included genes associated with inflammation, immunity, the CTLA4 pathway, oncogenes, or found to discriminate melanoma versus normal in previous exploratory studies [12]. Genes that discriminate between melanomas and healthy normals were determined by transcriptome profiling with microarray analysis identifying 78 such genes from which quantitative PCR confirmed the 27 melanoma associated genes included in the 169 gene panel $[12,14]$. Additional file 1 lists the full gene names and aliases.

Sample processing procedures were as described [12]. Whole-blood was collected in PAXgene ${ }^{\circledR}$ tubes and processed to RNA that met quality and integrity standards (RNA integrity number $\geq 6.3$ ) per the Bioanalyzer 2100 in combination with the RNA 6000 Nano or Pico Series II LabChip. First-strand complementary DNA was synthesized from random hexamer-primed RNA templates using $\operatorname{TaqMan}^{\circledast}$ reverse-transcription reagents. Individual target-gene amplification was multiplexed with the $18 \mathrm{~S}$ rRNA endogenous control and run in triplicate in 384-well format on the 7900HT fast real-time PCR system.

\section{Statistical analysis}

We tested the hypothesis in the discovery dataset and validated it in the independent validation dataset. Genes in both datasets are highly correlated and contain both predictors and "enhancer" variables, as first defined by Horst [15]. An enhancer variable, while not itself predictive of the outcome, is highly correlated with individual genes that are predictive of the criterion. Given technical variability, we eliminated low-expressing genes. With each heating/cooling cycle, the real time PCR assay detects fluorescent signal accumulation. The $\mathrm{Ct}$ (cycle threshold) is the number of cycles required for the fluorescent signal to exceed background level. Thermal cycling goes through 40 cycles, and $18 \mathrm{~S}$ rRNA is measured at the 14th cycle. Delta Ct is measured 26 cycles from the point endogenous control is recognized.

As part of our Quality Control program, mRNA transcript sets were assessed for technical variations using different reagent batches, operators and instruments. Technical variation for genes with delta $\mathrm{Ct}$ of $\leq 20$ was under 0.25 and increased to 0.5 when delta Ct was 20 to 25. Delta Ct of 23 was selected as a weak-reactions cutoff.

All pre-treatment genes in the 169-gene panel were assessed by the analysis of variance (ANOVA) t-test and the Mann-Whitney U Test for Unknown Distributions 
to determine predicators with a $p$ value $<0.05$. Both ANOVA $t$ and Mann-Whitney $U$ test results were determined for all individual predictor genes. Candidate synergistic 2-gene pairs included either predictors or a predicator plus an enhancer variable.

We used Statistical Innovations' CORExpress 1.1 commercial software, which employs correlated component regression analysis and handles multicolinearity due to correlated predictors effectively even with highdimensional data. The software was run in two-component mode for synergistic gene pair analysis. The final list of synergistic gene pairs, or "2-gene core models," was trained to predict response in the discovery dataset, tested next in the validation dataset based on objective response and then tested using one-year survival as the criterion. Over 260 pre-treatment response-predictive 2-gene core models were validated for objective response and survival.

Larger, optimal pre-treatment classifier models were constructed by combining validated 2-gene core models using CORExpress' correlated component regression package. The software was run in three-component, step-down mode starting with validated pre-treatment core models to eliminate weaker genes. At each step, resulting classifier models were validated for response and survival on the validation dataset. The discovery dataset's AUC was checked with publically-available MedCalc version 17 ROC analysis and $p$-value software [16]. The use of the CORExpress ${ }^{\circ}$ software handles multitesting, also called high-dimensional data, in which the number of potential predictors exceeds the number of test samples. "CORExpress 1.1 implements Correlated Component Regression (CCR) and focuses on regression analysis with a large numbers of correlated predictors $P$ which may exceed the sample size $n$ " Additional details can be obtained at the following website: http://www.sta tisticalinnovations.com/shop/corexpress/.

After development of a 15-gene predictive model for response using the discovery pre-treatment dataset and this methodology, the 15-gene algorithm was tested for both prediction of response and survival using the pretreatment validation dataset. Subsequently, the 15-gene pre-treatment algorithm was tested for response and 1-year survival using both the discovery and validation posttreatment datasets. One year survival was assessed as 1-year survival outcome data was available and provides early timepoint to assess the ability of the gene signature to discriminate for survival outcome but may not fully reflect multi-year survivial as with another CTLA-4 inhibitor, ipilimumab, longer term survival plateaus at approximately 3 -years. The step-wise statistical analysis utilized is outlined in Table 2.

The gene expression of components of the pretreatment gene signature was also compared in discovery dataset responders and nonresponders to expression in a
Table 2 Algorithm of the stepwise statistical analysis perfomed on the discovery and validation datasets

Step-Wise Statistical Analysis Using Discovery/Nalidation Methodology

Step 1: 2-Gene Models to Predict Immunotherapy Response and Survival

CORExpress 1.1 regression analysis software for high-dimensional data

Train 2-gene models with pre-treatment Discovery dataset $N=210$

Test 2-gene models with pre-treatment Validation dataset $N=150$

Over 260 2-gene synergistic pre-treatment models trained and validated

Step 2: Larger Gene Models to More Accurately Predict Response and Survival

Include only genes validated in 2-gene models from Step 1

Optimize model coefficients using CORExpress 1.1 software

Train optimized models with pre-treatment Discovery dataset $N=210$

Test optimized models with pre-treatment Validation dataset $N=150$

Step 3: Finalize 15-Gene Model to Predict Response and Survival

Optimal 15-gene pre-treatment model selected from Step 3

Use MedCalc version 17 software for ROC and $p$-value analysis

Test 15-gene model with pre-treatment Discovery dataset $N=210$

Test 15-gene model with pre-treatment validation dataset $N=150$

Step 4: Test Pre-treatment 15-Gene Model with Post-Treatment Datasets

15-gene pre-treatment response and survival model from Step 3

Use MedCalc version 17 software for ROC and $p$-value analysis

Test 15-gene model with post-treatment Discovery dataset $N=210$

Test 15-gene model with post-treatment Validation dataset $N=150$

set of 50 blood bank healthy normal volunteers. The Healthy Normal volunteers were $N=25$ female and $N=25$ male [14].

\section{Results}

Our goal was to identify peripheral blood-based biomarkers that predict response and one year survival to tremelimumab using blood samples collected before treatment initiation. Samples were available for 210 discovery dataset patients. The phase III trial was chosen as the discovery dataset because it required patients to be treatment-naïve, whereas patients in the phase II trial had received prior chemotherapy. Response was assessed 10 months after tremelimumab initiation. The treatment responses seen in both studies were mostly partial and in our analysis patients were identified as being a responder or a nonresponder. Using correlated component regression analysis we identified a 15-gene pre-treatment classifier model as optimal in terms of AUC for the discovery dataset. The 15-gene pre-treatment model consists of 9 predictors and six non-predictive enhancer variables, as illustrated in Fig. 1a. 


\begin{tabular}{|c|c|c|c|c|c|}
\hline \multicolumn{6}{|c|}{ PRE-TREATMENT DISCOVERY DATA SET N=210 } \\
\hline Gene & $\begin{array}{c}\text { ANOVA } \\
\text { t-test } \\
\text { p value }\end{array}$ & $\begin{array}{c}\text { Mann } \\
\text { Whitney } \\
\text { U Test } \\
\text { p value }\end{array}$ & Gene & $\begin{array}{l}\text { ANOVA } \\
\text { t-test } \\
\text { p value }\end{array}$ & $\begin{array}{c}\text { Mann } \\
\text { Whitney } \\
\text { U Test } \\
\text { p value }\end{array}$ \\
\hline \multicolumn{3}{|c|}{ PREDICTORS } & \multicolumn{3}{|c|}{ ENHANCER VARIABLES } \\
\hline CDK2 & 0.041 & 0.022 & ADAM17 & 0.101 & 0.113 \\
\hline CDKN2A & 0.017 & 0.010 & HLA-DRA & 0.607 & 0.953 \\
\hline DDP4 & 0.026 & 0.046 & MYC & 0.303 & 0.406 \\
\hline ERBB2 & 0.038 & 0.039 & RHOC & 0.381 & 0.538 \\
\hline ICOS & 0.020 & 0.040 & TGFB1 & 0.299 & 0.315 \\
\hline ITGA4 & 0.002 & 0.002 & TIMP1 & 0.206 & 0.101 \\
\hline LARGE & $>0.001$ & $>0.001$ & \multirow{3}{*}{\multicolumn{3}{|c|}{$\begin{array}{l}\text { Response-predictors } p \text { values are all smaller } \\
\text { than } 0 \cdot 05 \text {. Enhancer variables are not } \\
\text { response-predictive as individual genes. }\end{array}$}} \\
\hline NAB2 & 0.008 & 0.007 & & & \\
\hline NRAS & 0.028 & 0.030 & & & \\
\hline
\end{tabular}

C

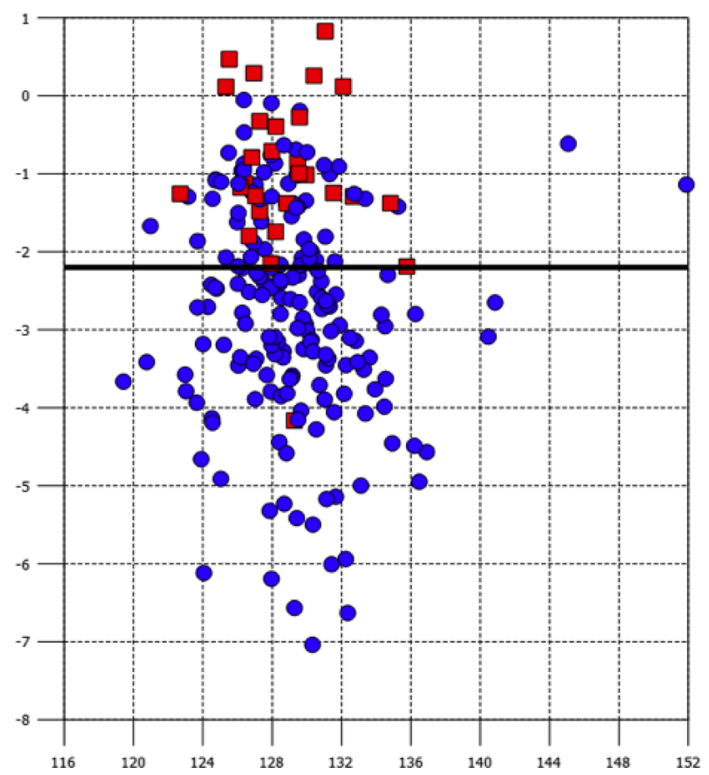

b

\begin{tabular}{|c|c|c|c|c|}
\hline & \multicolumn{4}{|c|}{$\begin{array}{c}\text { Pre-Treatment Blood-Based } \\
\text { 15-Gene mRNA Signature }\end{array}$} \\
\cline { 1 - 1 } $\begin{array}{c}\text { Melanoma } \\
\text { Cases }\end{array}$ & $\begin{array}{c}\text { Discovery } \\
\mathrm{N}=\mathbf{2 1 0}\end{array}$ & $\begin{array}{c}\text { Discovery } \\
\mathrm{N}=210\end{array}$ & $\begin{array}{c}\text { Validation } \\
\mathrm{N}=150\end{array}$ & $\begin{array}{c}\text { Validation } \\
\mathrm{N}=150\end{array}$ \\
\hline Biomarker & Response & Survival & Response & Survival \\
\hline Sensitivity & $96.4 \%$ & $51.7 \%$ & $65.0 \%$ & $76.7 \%$ \\
\hline Specificity & $65.9 \%$ & $69.6 \%$ & $59.2 \%$ & $57.0 \%$ \\
\hline NPV & $99.2 \%$ & $59.2 \%$ & $91.7 \%$ & $85.9 \%$ \\
\hline AUC & 0.862 & 0.605 & 0.622 & 0.676 \\
\hline P value & $<0.0001$ & 0.0066 & 0.0455 & 0.0002 \\
\hline
\end{tabular}

d

\begin{tabular}{|c|c|c|c|c|}
\hline & \multicolumn{4}{|c|}{$\begin{array}{c}\text { Post-Treatment Blood-Based } \\
\text { 15-Gene mRNA Signature }\end{array}$} \\
\hline $\begin{array}{c}\text { Test Data } \\
\text { Sets } \\
\text { Melanoma } \\
\text { Cases }\end{array}$ & $\begin{array}{c}\text { Discovery } \\
\text { Post- } \\
\text { Treatment } \\
\text { Dataset } \\
\text { N=210 }\end{array}$ & $\begin{array}{c}\text { Discovery } \\
\text { Post- } \\
\text { Treatment } \\
\text { Dataset } \\
\text { N=210 }\end{array}$ & $\begin{array}{c}\text { Validation } \\
\text { Post- } \\
\text { Treatment } \\
\text { Dataset } \\
\text { N=150 }\end{array}$ & $\begin{array}{c}\text { Validation } \\
\text { Post- } \\
\text { Treatment } \\
\text { Dataset } \\
\mathrm{N}=150\end{array}$ \\
\hline Biomarker & Response & Survival & Response & Survival \\
\hline Sensitivity & $\mathbf{5 7 . 1 \%}$ & $\mathbf{3 0 . 5 \%}$ & $\mathbf{9 0 . 0 \%}$ & $\mathbf{8 3 . 7 \%}$ \\
\hline Specificity & $\mathbf{7 9 . 7 \%}$ & $\mathbf{8 8 . 4 \%}$ & $33.1 \%$ & $41.1 \%$ \\
\hline NPV & $90.2 \%$ & $49.7 \%$ & $95.6 \%$ & $\mathbf{8 6 . 3 \%}$ \\
\hline AUC & $\mathbf{0 . 6 8 4}$ & 0.588 & 0.562 & 0.619 \\
\hline p Value & 0.0016 & 0.0246 & 0.3253 & 0.0140 \\
\hline
\end{tabular}

Fig. 1 The 15-gene signature predicting response in the pre-treatment discovery data set consists of 9 predictor genes and 6 enhancer variable genes (a). The sensitivity, specificity, negative predictive value, area under the curve and p-value of the pre-treatment 15 gene signature predicting response in the discovery data set and both response and survival in the validation dataset (b). Composite response-prediction score generated to visually represent responders versus non responders. $X$ Axis is the correlated component score and $Y$ axis is the composite response-prediction score. Red squares (responders) and blue circles (non-responders) (c). Testing of the post-treatment discovery and validation datasets prediction of response and survival including the sensitivity, specificity, negative predictive value, area under the curve and p-value using the pre-treatment 15 gene signature (d)

The 15-gene pre-treatment model is represented by the formula:

Predicted pre-treatment to respond to tremelimumab $>24.009-(0.8697 x$ ADAM17) $+(0.7486 x$ CDK2) $-(0.5885 x \mathrm{xD}$ $\mathrm{KN} 2 \mathrm{~A})+(0.3462 \mathrm{xDPP} 4)-(0.2401 \times \mathrm{xEBB} 2)+(1.7427 \mathrm{xHL}$ A-DRA $)+(0.2481 x I C O S)-(1.1975 x I T G A 4)-(1.0184 x L A R$ GE) + (1.1721xMYC)-(0.6531xNAB2)-(1.1491xNRAS) + (0.7377xRHOC)-(1.0585xTGFB1) + (0.8328xTIMP1).

As Fig. 1b illustrates the model predicted response with a negative predictive value (NPV), $\mathrm{z}$ statistic and AUC, of 99.2\%, 10.1, and 0.862 (95\% confidence interval 0.807 to $0.905, p<0.0001$ ), respectively. The model predicted one-year survival with a NPV, $\mathrm{z}$ statistic and AUC, of 59.2\%, 2.72, and 0.605 (95\% confidence interval 0.536 to $0.672, p=0.0066$ ), respectively. The model was applied subsequently to the independent validation dataset of 150 melanoma patients. The genes, constant and gene coefficients remained the same as in the discovery model and a fixed cutoff was used. The validation dataset NPV, z statistic, AUC were 91.7\%, 2.0, and 0.622 (95\% confidence interval 0.539 to $0.699, p=0.0455)$, respectively, thereby validating the model's ability to predict response (Table 2b). The pre-treatment model was also predictive of one-year survival with a NPV of $85.9 \%, \mathrm{z}$ statistic of 3.7, and AUC of 0.676 ( $95 \%$ confidence interval 0.594 to $0.750, p=0.0002$ ). The composite pre-treatment response prediction scores demonstrated clear differentiation between responders and non-responders (Fig. 1c).

As Fig. 1d illustrates the 15-gene pre-treatment model also predicted response using the discovery post-treatment 
dataset with a NPV, z statistic and AUC, of 92.4\%, 3.2, and 0.684 (95\% confidence interval 0.616 to $0.746, p=0.0016$ ), respectively. The model also predicted survival in the posttreatment dataset with a NPV, z statistic and AUC, of 49.7\%, 2.25, and 0.588 (95\% confidence interval 0.518 to $0.655 p=0.0246)$, respectively. The model did not predict for response using the validation post-treatment dataset with a NPV, z statistic and AUC, of 95.6\%, 0.98, and 0.592 (95\% confidence interval 0.478 to 0.642 , and $p=0.3253$ ), respectively. The model, however, predicted survival in the validation post-treatment dataset with a NPV, z statistic and AUC, of $86.3 \%, 2.6$, and 0.619 (95\% confidence interval 0.536 to 0.697 , and $p=0.0140$ ), respectively.

While the 15-gene signature predicted for response and one year survival, many other genes were up-regulated in a statistically significant manner in responders versus non-responders in the phase III trial. However, these same genes showed no difference in responders versus nonresponders in the validation phase II trial. CTLA4, for example, showed a statistically significant pre-treatment response-predictive ANOVA t-test ( $p$ value $=0.005)$ in the phase III but not the phase II where the ( $p$ value $=0.346$.) Table 3 shows eight examples of phase III pre-treatment response-predictors that are not statistically significant in the phase II study.

The data highly suggest that the expression of the 15genes in the signature represent expression levels of particular genes needed for robust immune responses against cancer. Expression of these genes may identify patients whose immune systems are already primed to have an anti-cancer immune response. Therefore the level at which the 15 genes were expressed in discovery dataset responders was compared to expression in a set of 50 blood bank healthy normal volunteers. Unexpectedly only 6 genes demonstrated differential expression being either up or down regulated when responders were compared to healthy normal controls of which 5 of these 6 genes were enhancer genes. Eight of the genes

Table 3 Examples of genes predictive for response in the discovery but not validation datasets

\begin{tabular}{lll}
\hline Gene & $\begin{array}{l}N=210 \text { Discovery } \\
\text { Pre-treatment } \\
\text { ANOVA t-test }\end{array}$ & $\begin{array}{l}N=150 \text { Validation } \\
\text { Pre-treatment } \\
\text { ANOVA t-test }\end{array}$ \\
\hline CD28 & 0.026 & 0.158 \\
CD80 & 0.012 & 0.368 \\
FAIM3 & 0.008 & 0.638 \\
FYN & 0.006 & 0.962 \\
IL18BP & 0.020 & 0.958 \\
IL32 & 0.021 & 0.686 \\
IL7R & 0.009 & 0.590 \\
INPP4B & 0.006 & 0.740 \\
\hline
\end{tabular}

demonstrated equivalent expression between responders and healthy normal controls (Table 4) of which 7 of these 8 genes were predictor genes.

As discussed above, the 15-gene signature contains 6 enhancer variable genes which do not independently predict response but rather enhance the predictive ability of the 9 predictor genes. While gene expression of most of the enhancers differed between responders and healthy normals only 1 (potentially 2 ) of the 9 predictive genes demonstrated differential expression (ICOS is a predictive gene but its mRNA expression was not available for measurement in the healthy normals).

Given that almost all of the predictor genes showed equivalent expression in responders and healthy normals we hypothesized that the predictor genes were differentially expressed in the non-responders. As shown in Table 4 all eight evaluable predictive genes were in fact down-regulated in the non-responders relative to responders. However all six enhancers showed no significant change in gene expression between responders and non-responders.

\section{Discussion}

An ideal biomarker should be obtained easily with minimal risk to the patient. Biomarkers based on mRNA transcript gene expression profiling obtained from whole blood have enormous advantages over tumor-based gene expression profiling. Tumor biopsies are invasive and difficult to obtain. Blood samples are much less invasive and less costly to obtain, have minimal risk, can be serially obtained, and are collectible by non-physicians. Potential biomarkers of efficacy following CTLA-4 blockade have been reported based on characteristics present in peripheral blood, including T-lymphocyte ICOS expression, neutrophil/lymphocyte ratio, CTLA-4 polymorphisms, effector/suppressor T-lymphocyte ratio, absolute lymphocyte and eosinophil counts, T-cell receptor diversity, and nomogram model score comprising baseline lactate dehydrogenase value and absolute neutrophil count [17]. These markers need validation.

The pretreatment gene signature we identified and validated contains 9 predictor genes. The remaining 6 genes are enhancer variable genes which do not individually predict for response but rather enhance the predictive ability of the predictors. Use of enhancer variables decreases extraneous variation and strengthens the relationship between a predictor and a given criterion (response rate or survival). Understanding the function of the predictive genes can help identify cellular processes associated with benefit following inhibition of CTLA-4 activity.

Given that tremelimumab is immunomodulatory, one might hypothesize that a gene expression signature predicting efficacy would encompass predominately genes directly modulating immune activity or the tremelimumab 
Table 4 Relative gene expression of the 15 genes comprising the pre-treatment signature comparing responders in the discovery dataset to healthy volunteers and to non-responders

\begin{tabular}{|c|c|c|c|c|c|c|}
\hline \multirow{3}{*}{$\begin{array}{l}\text { 15-Gene Pre-Treatment } \\
\text { Model }\end{array}$} & \multirow{3}{*}{$\begin{array}{l}\text { Predictor or } \\
\text { Enhancer Variable }\end{array}$} & \multirow{3}{*}{$\begin{array}{l}\text { Blood Bank } \\
N=50 \\
\text { Healthy Normals }\end{array}$} & \multirow{3}{*}{$\begin{array}{l}\text { Difference Normals } \\
\text { versus Responders }\end{array}$} & \multicolumn{2}{|c|}{ Phase 3 Discovery Dataset } & \multirow{3}{*}{$\begin{array}{l}\text { Difference } \\
\text { Responders vs } \\
\text { Non-responders }\end{array}$} \\
\hline & & & & \multirow{2}{*}{$\begin{array}{l}N=28 \\
\text { Responders }\end{array}$} & \multirow{2}{*}{$\begin{array}{l}N=182 \\
\text { Non-Responders }\end{array}$} & \\
\hline & & & & & & \\
\hline \multicolumn{7}{|c|}{ Responders Equivalent to Normals } \\
\hline ITGA4 & Predictor & 14.2 & 0.02 & 14.22 & 14.61 & 0.39 \\
\hline LARGE & Predictor & 22.0 & 0.09 & 22.09 & 22.97 & 0.88 \\
\hline CDK2 & Predictor & 19.6 & 0.09 & 19.69 & 19.91 & 0.22 \\
\hline TIMP1 & Enhancer & 15.0 & 0.10 & 15.1 & 14.95 & -0.15 \\
\hline DPP4 & Predictor & 18.5 & 0.12 & 18.62 & 18.95 & 0.33 \\
\hline NRAS & Predictor & 17.1 & 0.13 & 17.23 & 17.44 & 0.21 \\
\hline ERBB2 & Predictor & 23.0 & -0.18 & 22.82 & 23.23 & 0.41 \\
\hline NAB2 & Predictor & 20.0 & -0.29 & 19.71 & 20.04 & 0.33 \\
\hline \multicolumn{7}{|c|}{ Responders Upregulated Compared to Normals } \\
\hline ADAM17 & Enhancer & 18.5 & 0.32 & 18.18 & 18.36 & 0.18 \\
\hline $\mathrm{RHOC}$ & Enhancer & 16.9 & 0.39 & 16.51 & 16.63 & 0.12 \\
\hline TGFB1 & Enhancer & 13.4 & 0.45 & 12.95 & 13.05 & 0.10 \\
\hline CDKN2A & Predictor & 21.4 & 0.63 & 20.77 & 21.16 & 0.39 \\
\hline \multicolumn{7}{|c|}{ Responders Downregulated Compared to Normals } \\
\hline HLADRA & Enhancer & 12.1 & 0.48 & 12.58 & 12.64 & 0.06 \\
\hline MYC & Enhancer & 17.7 & 0.82 & 18.53 & 18.67 & 0.14 \\
\hline \multicolumn{7}{|c|}{ Measurement of Gene Expression Not Available } \\
\hline ICOS & Predictor & N/A & N/A & 22.32 & 22.78 & 0.46 \\
\hline
\end{tabular}

target CTLA-4. However only two of the nine predictor genes (ICOS and DPP4) are direct modulators of immune activity. ICOS is an inducible co-stimulatory molecule expressed on active CD4+ T-lymphocytes [18]. DPP4, a dipeptidyl peptidase subfamily member of serine proteases, regulates chemokine activity [19]. Baseline gene expression of the tremelimumab target CTLA-4 is not a component of the signature and not a validated predictive gene.

However the immune milieu of the tumor microenvironment could be modulated through complex mechanisms which facilitate responsiveness to CTLA-4 inhibition. Activation of signal transduction pathways such as the MAPK pathway and changes to extracellular components can alter the immune activity in tumor microenvironments. Five of the predictor genes are components of signal transduction pathways or regulate cell cycle progression. The genes associated with signal transduction affect multiple pathways but the MAPK and PI3K/AKT pathways in particular. NRAS regulates signal transduction pathway activity, including MAPK and AKT pathways. ERBB2 dimerizes with EGFR, modulating downstream ERK and AKT signaling [20] NAB2 regulates mRNA transcripts targeting for export through the nuclear pore [21].
The genes directly associated with cell cycle progression regulate different phases of the cycle. CDKN2A encodes p16, a tumor suppressor that binds CDK4/6 inhibiting cyclin-dependent kinase activity regulating the $\mathrm{G} 1$ portion of the cycle [22]. CDK2 is a cyclin-dependent kinase that regulates mitotic entry [23]. Two other predictor genes regulate extracellular matrix integrity (LARGE, a glycosyltransferase with substrates involved in cellular connection to basement membranes [24] and ITGA4, an integrin regulating cell adhesion to the extracellular matrix). Therefore, the combined mRNA expression of key signal transduction pathway components and regulators of the extracellular tumor microenvironment in combination with certain direct regulators of immune activity (ICOS, DPP4) appears to predict priming for responsiveness to CTLA-4 blockade.

We found that relative to healthy normal controls expression of the response predictive genes was preserved in responders but significantly down regulated in nonresponders. The healthy controls were not matched to the patients included in our analysis by clinical features that can modulate immune activity such as age or gender. As such while the findings are hypothesis generating we cannot conclude that preserved expression of these 
genes in a patient's whole blood mRNA reflects the ability of a patient's melanoma to escape immune destruction by tremelimumab.

Another limitation of the study is that patients treated in the discovery cohort were treatment naïve while patients treated in the validation cohort received prior treatment with chemotherapy. Prior chemotherapy exposure could potentially alter subsequent responsiveness to tremelimumab, the expression level of genes evaluated, and the degree of predictiveness that the gene signature identified. As noted above, the treatment-naïve discovery population had longer median and greater one-year survival than the chemotherapy-refractory validation population. The sicker validation patient population might explain why many promising biomarkers (e.g., pretreatment CTLA4 gene expression) were up-regulated in responders versus non-responders to a statistically significant degree in the phase III but not the phase II trial. If both the discovery and validation datasets were treatmentnaive or not refractory to cytotoxic chemotherapy, additional response-predictive biomarkers would likely be identified. The treatment responses seen in both studies were mostly partial. In our analysis patients were identified as being a responder or a nonresponder. While the gene signature identified predicts for response we cannot conclude that it predicts for the degree of response.

The gene signature reported here is predictive and prognostic in the context of CTLA-4 inhibition with tremelimumab specifically. A limitation of the clinical application of the identified gene signature is that tremelimumab is not an FDA approved treatment for melanoma. Rather the CTLA-4 inhibitor ipilimumab is approved and while both antibodies inhibit CTLA-4 they are not necessarily equivalent in mechanism of action and predictive gene signatures may differ. Our analysis included only patients with both pre- and post- treatment blood samples. While this includes the majority of patients enrolled in the discovery and validation studies a limitation of our analysis is that not all patients treated in both studies were included. However a gene signature was identified and validated with this subset of patients and the discovery in the blood of a predictive gene signature provides a proof of concept. A similar strategy can be used to identify gene signatures in the context of treatment with other immune modulators including ipilimumab and anti-PD1 inhibitors.

Currently, few melanoma patients are treated with cytotoxic chemotherapy before receiving CTLA- 4 and PD-1 inhibitors. Patients whose melanoma expresses V600 mutated BRAF may receive therapy with BRAF and MEK inhibitors. Often, single agent PD-1 inhibitor or combination ipilimumab plus nivolumab therapy is chosen first line. Phase III comparison of 834 stage IV melanoma patients randomized to initial treatment with pembrolizumab versus ipilimumab demonstrated significant improvement in response rate and 12 month survival, favoring PD-1 inhibitor treatment [7]. The effects of prior BRAF/MEK inhibitor, or PD-1 blockade therapy on the gene signature robustness is unknown. Presence of a transcriptional signature termed IPRES (innate anti-PD-1 resistance) in melanoma biopsy specimens was enriched for patients resistant to PD-1 inhibition but did not predict response to anti-CTLA-4 therapy [25]. Clinical trials using blood-based mRNA classifier models are warranted for PD-1 inhibitors and combinations of CTLA-4 plus PD-1 inhibitors, as their distinct targets and clinical efficacy rates differ.

This is the first large clinical study that has been validated independently in a second large clinical study to show that a response-predictive mRNA signature can be documented in blood prior to tremelimumab treatment. The signature demonstrates the potential of pre-treatment mRNA expression profiles derived from blood to predict clinical benefit. Predictive model components regulate immune activity, cell cycle proliferation, and extracellular matrix composition. Using such models can help to optimize the efficacy and safety of therapy for patients with advanced melanoma. Moreover, this strategy can be applied to PD-1 blockade and to other third generation immunomodulatory treatments currently in development.

\section{Conclusions}

Our study demonstrates that pretreatment expression of a signature of 15 genes derived from whole blood samples obtained from patients with stage IV melanoma can predict for response to a CTLA-4 inhibitor. This allows for a minimally invasive way to identify prospectively responders to treatment. This information would optimize treatment planning allowing patients unlikely to respond to receive alternative treatments sooner, minimize toxicity risk, and allow for more efficient utilization of health care spending. Future research will need to utilize a similar approach to identify blood-based biomarkers predictive pretreatment of efficacy to anti-PD-1 based and other checkpoint inhibitor combination therapies.

\section{Additional file}

\section{Additional file 1: List of Genes with Full Name and Aliases of Each.} (DOCX $33 \mathrm{~kb})$

\section{Abbreviations}

AUC: Area under the curve; Ct: Cycle threshold; CTLA-4: Cytotoxic

T-lymphocyte associated protein 4; FDA: Food and drug administration; IHC: Immunohistochemistry; N: Number; NPV: Negative predictive value; PCR: Polymerase chain reaction; PD-1: Programmed death-1; PD-L1: Programmed death-ligand 1; RNA: Ribonucleic acid; RRNA: Ribosomal ribonucleic acid 


\section{Acknowledgements}

We thank Dr. Steven Burakoff, Dr. Matthew Galsky, and Dr. Eric Schadt for their contributions to this work.

\section{Funding}

This study was funded by CPS Companion Diagnostics.

\section{Availability of data and materials}

De-identified datasets used and/or analyzed during the current study are available from author $\mathrm{KW}$ upon reasonable request.

\section{Authors' contributions}

Study conception and design: PF, KW, NB, DF, WKO. Gene expression model: KW. Statistical analyses: KW. Drafting of manuscript: PF, KW, AMC, CK. Critical revision of manuscript: all authors. All authors read and approved the final manuscript.

\section{Ethics approval and consent to participate}

The current submission does not need separate ethical approval. The study presented in this manuscript analyzes RNA obtained from blood of patients enrolled in two different clinical trials sponsored by Pfizer. RNA analysis of the Pfizer study data was contemplated in the original protocol consents. Therefore this study does not need ethical approval as the subjects had to consent to participate in the two studies and agree to have their blood drawn and their gene expression analyzed. We further were totally blinded as to the patient's identifiers with HIPPA protected data.

\section{Consent for publication}

\section{Not applicable}

\section{Competing interests}

PF: Advisory Board Member for EMD Serono and Castle Biosciences. KW: President and CEO of CPS Companion Diagnostics. AMC: Chairman of CPS Companion Diagnostics. DF: The author declares that he has no competing interests. CK: The author declares that she has no competing interests. JMK: Consultant for BMS, Amgen, Green Peptide, Roche, Solaran RX, Checkmate Pharmaceuticals and Novartis. NB: Consultant for Neon. Advisory Board Member for CPS Companion Diagnostics and Curevac. WKO: Chief Scientific Officer and Chairman of the Science Advisory Board CPS Companion Diagnostics.

\section{Publisher's Note}

Springer Nature remains neutral with regard to jurisdictional claims in published maps and institutional affiliations.

\section{Author details}

'Division of Hematology and Medical Oncology, Tisch Cancer Institute, Icahn School of Medicine at Mount Sinai, Mount Sinai Hospital, New York, NY, USA. ${ }^{2}$ CPS Companion Diagnostics, Cambridge, MA, USA. ${ }^{3}$ Department of Dermatology, Harvard Medical School, Boston, MA, USA. ${ }^{4}$ Departments of Medicine, Dermatology and Translational Sciences, University of Pittsburgh School of Medicine, Pittsburgh, PA, USA. ${ }^{5}$ Parker Institute of Cancer Immunotherapy, San Francisco, CA, USA.

Received: 4 May 2017 Accepted: 1 August 2017 Published online: 15 August 2017

\section{References}

1. Hodi FS, O'Day SJ, McDermott DF, Weber RW, Sosman JA, Haanen JB, Gonzalez R, Robert C, Schadendorf D, Hassel JC, et al. Improved survival with ipilimumab in patients with metastatic melanoma. N Engl J Med. 2010; 363(8):711-23.

2. Schadendorf D, Hodi FS, Robert C, Weber JS, Margolin K, Hamid O, Patt D, Chen TT, Berman DM, Wolchok JD. Pooled analysis of long-term survival data from phase II and phase III trials of ipilimumab in unresectable or metastatic melanoma. J Clin Oncol. 2015;33(17):1889-94.

3. Kirkwood JM, Lorigan P, Hersey P, Hauschild A, Robert C, McDermott D, Marshall MA, Gomez-Navarro J, Liang JQ, Bulanhagui CA. Phase II trial of tremelimumab (CP-675,206) in patients with advanced refractory or relapsed melanoma. Clin Cancer Res. 2010;16(3):1042-8.
4. Ribas A, Kefford R, Marshall MA, Punt CJ, Haanen JB, Marmol M, Garbe C, Gogas H, Schachter J, Linette G, et al. Phase III randomized clinical trial comparing tremelimumab with standard-of-care chemotherapy in patients with advanced melanoma. J Clin Oncol. 2013;31(5):616-22.

5. Weber JS, D'Angelo SP, Minor D, Hodi FS, Gutzmer R, Neyns B, Hoeller C, Khushalani NI, Miller WH Jr, Lao CD, et al. Nivolumab versus chemotherapy in patients with advanced melanoma who progressed after anti-CTLA-4 treatment (CheckMate 037): a randomised, controlled, open-label, phase 3 trial. Lancet Oncol. 2015;16(4):375-84.

6. Ribas A, Puzanov I, Dummer R, Schadendorf D, Hamid O, Robert C, Hodi FS, Schachter J, Pavlick AC, Lewis KD, et al. Pembrolizumab versus investigatorchoice chemotherapy for ipilimumab-refractory melanoma (KEYNOTE-002): a randomised, controlled, phase 2 trial. Lancet Oncol. 2015;16(8):908-18.

7. Robert C, Schachter J, Long GV, Arance A, Grob JJ, Mortier L, Daud A, Carlino MS, McNeil C, Lotem M, et al. Pembrolizumab versus Ipilimumab in advanced melanoma. N Engl J Med. 2015;372(26):2521-32.

8. Larkin J, Hodi FS, Wolchok JD. Combined nivolumab and ipilimumab or monotherapy in untreated melanoma. N Engl J Med. 2015;373(13):1270-1.

9. Nisen M. Cancer drugs can't stay this expensive forever. BloombergGadfly. 2016. https://www.bloomberg.com/gadfly/articles/2016-06-13/cancer-drugscan-t-stay-this-expensive-forever

10. Jorgensen JT. Companion diagnostic assays for PD-1/PD-L1 checkpoint inhibitors in NSCLC. Expert Rev Mol Diagn. 2016;16(2):131-3.

11. Wolchok J, V Chiaron-Selini, Gonzalez R, Rutkowski P, Grob J, Cowey CL, Lao C, Schadendorf D, PF Ferrucci, M Smylie, R Dummer, A Hill, JBAG Haanen, M Maio, GA McArthur, D Walker, J Jiang, CE Horak, JMG Larkin, F Stephen Hodi. Updated results from a phase III trial of nivolumab (NIVO) combined with ipilimumab (IPI) in treatment-naive patients (pts) with advanced melanoma (MEL) (CheckMate 067). J Clin Oncol. 2016;34(suppl 15):9505.

12. Saenger Y, Magidson J, Liaw B, de Moll E, Harcharik S, Fu Y, Wassmann K, Fisher D, Kirkwood J, Oh WK, et al. Blood mRNA expression profiling predicts survival in patients treated with tremelimumab. Clin Cancer Res. 2014;20(12):3310-8

13. Therasse P, Arbuck SG, Eisenhauer EA, Wanders J, Kaplan RS, Rubinstein L, Verweij J, Van Glabbeke M, van Oosterom AT, Christian MC, et al. New guidelines to evaluate the response to treatment in solid tumors. European Organization for Research and Treatment of Cancer, National Cancer Institute of the United States, National Cancer Institute of Canada. J Natl Cancer Inst. 2000;92(3):205-16.

14. Luo Y, Robinson S, Fujita J, Siconolfi L, Magidson J, Edwards CK, Wassmann K, Storm K, Norris DA, Bankaitis-Davis D, et al. Transcriptome profiling of whole blood cells identifies PLEK2 and C1QB in human melanoma. PLoS One. 2011;6(6):e20971.

15. Horst P. The prediction of personal adjustment. Soc Science Res Council Bull. 1941;48:1-156.

16. ROC Analysis. Web-based calculator for ROC curves. www.rad.jhmi.edu/jeng/ javarad/roc/JROCFITi.html.

17. Friedman CF, Postow MA. Emerging tissue and blood-based biomarkers that may predict response to immune checkpoint inhibition. Curr Oncol Rep. 2016;18(4):21.

18. Sanmamed MF, Pastor F, Rodriguez A, Perez-Gracia JL, Rodriguez-Ruiz ME, Jure-Kunkel M, Melero I. Agonists of co-stimulation in cancer immunotherapy directed against CD137, OX40, GITR, CD27, CD28, and ICOS. Semin Oncol. 2015;42(4):640-55.

19. Metzemaekers M, Van Damme J, Mortier A, Proost P. Regulation of chemokine activity - A focus on the role of dipeptidyl peptidase IV/CD26. Front Immunol. 2016;7:483.

20. Croucher DR, Iconomou M, Hastings JF, Kennedy SP, Han JZ, Shearer RF, McKenna J, Wan A, Lau J, Aparicio S, et al. Bimolecular complementation affinity purification (BiCAP) reveals dimer-specific protein interactions for ERBB2 dimers. Sci Signal. 2016:9(436):ra69.

21. Stewart M. Nuclear export of mRNA. Trends Biochem Sci. 2010;35(11):609-17.

22. Zhao R, Choi BY, Lee MH, Bode AM, Dong Z. Implications of genetic and epigenetic alterations of CDKN2A (p16(INK4a)) in cancer. EBioMedicine. 2016;8:30-9.

23. Hein JB, Nilsson J. Interphase APC/C-Cdc20 inhibition by cyclin A2-Cdk2 ensures efficient mitotic entry. Nat Commun. 2016;7:10975.

24. Yoshida-Moriguchi T, Campbell KP. Matriglycan: a novel polysaccharide that links dystroglycan to the basement membrane. Glycobiology. 2015;25(7):702-13.

25. Hugo W, Zaretsky JM, Sun L, Song C, Moreno BH, Hu-Lieskovan S, Berent-Maoz B, Pang J, Chmielowski B, Cherry G, et al. Genomic and transcriptomic features of response to anti-PD-1 therapy in metastatic melanoma. Cell. 2016;165(1):35-44. 